\title{
Book Review - Buchbesprechung - Livre nouveau
}

A. Jolly: The Evolution of Primate Behavior. Macmillan, New York 1972.

Vor allem Anthropologen, Primatologen, Ethologen und Psychologen werden Alison Jolly dankbar dafür sein, dass sie den nahezu heroischen Versuch unternommen hat, eine Übersicht über das gegenwärtige Wissen vom Verhalten der Primaten zu erarbeiten. Angesichts des rapiden Wachstums dieses Forschungsgebietes konnte dieser Versuch, wie die Verfasserin im Vorwort selber erklärt, grundsätzlich nur teilweise gelingen. Immerhin hat sie in diesem 400seitigen Buch über 500 einschlägige Veröffentlichungen berücksichtigt. Schon dieses Literaturverzeichnis, das von Yerkes bis Premack reicht, wird manchem Suchenden willkommen sein, zumal das Buch sehr klar gegliedert ist; seine drei Hauptteile sind Ecology, Society, Intelligence. Jedem der 20 Kapitel ist eine kurze Zusammenfassung beigefügt, sehr oft auch eine äusserst instruktive tabellarische Übersicht. Die rund 160 Illustrationen stammen aus anderen Veröffentlichungen.

Im Abschnitt über die Ernährung wird die Hypothese aufgestellt, dass die Abzweigung der Hominiden von anderen Primaten mit dem Essen von Sämereien (seed) angefangen haben könnte. Primaten haben wenig natürliche Feinde; die wichtigsten sind weder Raub-vögel noch Raubtiere, sondern andere Primaten. Schimpansen fangen, töten und essen gelegentlich Rote Colobus und junge Paviane; Paviane tun dasselbe mit Meerkatzen. Alarmrufe sind oft feindspezifisch (Luft-und Bodenfeinde); grüne Meerkatzen haben sogar einen besonderen Schlangen-Warnruf.

Ob Territorien gebildet und verteidigt werden, hängt nicht nur von ökologischen Fak-toren ab, sondern auch von der stammesgeschichtlichen Entwicklung. So haben weder die Menschenaffen noch die Vertreter der Makaken-Pavian-Linie verteidigte Territorien, wohl aber die der GuenonGruppe, gleichgültig ob sie im Wald oder in der Savanne leben.

Zwischen angeborenem und gelerntem Verhalten kann keine scharfe Trennung vor-genommen werden: «Any behavioral trait is both learned and innate.» Gelegentlich wird etwas rasch verallgemeinert, so gilt zum Beispiel nicht für alle Säugetier-Mutter, dass sie ihre Jungen lecken; Mutter vom passiven Typ, etwa Cameliden, tun das nicht.

Dem Sozialverhalten sind aufschlussreiche Darstellungen über Kommunikation, Status, Grooming, Mutter-Kind-Beziehung, Heranwachsen in der Gruppe u. a. gewidmet, sowie ein bemerkenswerter Abschnitt über die Tante (aunt). Dieser Figur kommt - nicht nur bei Primaten, sondern auch bei Huftieren, Elefan ten, Walen us w. nach neueren Beobachtungen eine soziologisch wichtige Funktion zu. Es handelt sich dabei nicht um eine Blutsverwandte, sondern um ein nahestehendesWeibchen, das an der Betreuungeines fremden Neugebore-nen ein aktives Interesse nimmt und dabei die Mutter unterstützt und entlastet. Jolly gibt eindrucksvolle Beispiele von solchem « aunt-behavior » von alt- und neuweltlichen Primaten und auch von den der Autorin besonders vertrauten Lemuren.

Die Kapitel des 3. Hauptteiles (Intelligenz) behandeln Psychologie, Werkzeuggebrauch, Kognition, Sprache, soziales Lernen und die Evolution der Intelligenz. Damit ist erneut angedeutet, wie ungeheuer weit das von Jolly untersuchte Forschungsgebiet ist. Einem 
einzelnen Autor wird es kaum möglich sein, eine bessere und umfassendere Übersicht zu bieten als die vorliegende. Für den Leser, der sich mit der Evolution des Primatenverhaltens vertraut machen möchte, ist sie eine wertvolle Einführung, für den Spezialisten eine unerlässliche Ergänzung. H. Hediger, Zurich 